\title{
Modelagem matemática para a distribuição física de produtos da cadeia do Frio
}

Gabriel Alexandre Müller gabriel_goetz@hotmail.com Universidade Federal de Santa Catarina (UFSC), Joinville, SC, Brasil

Vanina Macowski Durski Silva

vanina.durski@ufsc.br

Universidade Federal de Santa Catarina (UFSC), Joinville, SC Brazil

\begin{abstract}
RESUMO
No intuito de preservar a qualidade de produtos que requerem monitoramento de temperatura desenvolvem-se métodos e estudos sobre a Cadeia do Frio, a qual é responsável pela cadeia logística do produto, desde sua produção, armazenamento até a distribuição física ao consumidor final. Neste contexto, este artigo busca compreender o comportamento da variação de temperatura de cargas refrigeradas durante sua distribuição física e para isso, através da modelagem matemática implementam-se equações que representam os comportamentos de variação térmica durante a entrega de produtos, considerando os processos de carregamento, deslocamento e descarregamento dos mesmos. O objetivo deste estudo é, portanto, estudar este comportamento de modo a gerar uma ferramenta de simulação que seja capaz de planejar rotas de distribuição física de produtos refrigerados, tornando mais eficiente todo o processo logístico, reduzindo o ganho indesejado de temperatura interna do veículo e do produto.
\end{abstract}

PALAVRAS-CHAVE: Cadeia do frio. Modelagem matemática. Roteirização. Refrigeração. Distribuição. 


\section{INTRODUÇÃO}

O termo cadeia do frio tem sido usado para denominar os processos e elos de produção e logística de um produto refrigerado, com o objetivo de conservar e evitar o seu desperdício. O objetivo da cadeia do frio, conforme definida por Heap (2006), IIR (2004) e, Likar e Jevsnik (2006), compreende o controle da temperatura do produto em todas as fases de transporte e estocagem, até a entrega ao cliente final. Qualquer rompimento desta cadeia pode provocar a perda de qualidade do produto, pois se sabe que as velocidades de reações químicas, microbiológicas e bioquímicas perceptíveis nos produtos estão diretamente relacionadas à temperatura, portanto, é de importância considerável manter um controle adequado durante todos os processos envolvidos.

Tem-se que a perda de qualidade do produto é cumulativa e irreversível (Heap, Kierstan e Ford, 1998), e com isso surge a necessidade de conhecer e respeitar seus limites de temperatura. Quando o produto alcança uma determinada temperatura acima de seu limite superior pode sofrer diminuição de sua qualidade final, ocasionando sua rejeição. Porém, temperaturas abaixo do limite inferior suportado pelo produto também podem prejudicar suas características iniciais, cobrando uma complexidade logística maior no manuseio das mercadorias em estoque e transporte.

Segundo Carvalho (2013), a atividade logística de transporte é um processo crítico da cadeia do frio. A The Cool Chain Association (CCA) estima que 30\% dos produtos sensíveis à temperatura são perdidos durante este processo (HOFFMAN, 2006). Sendo assim, este artigo propõe-se a analisar e compreender um modelo matemático que represente comportamentos térmicos de um processo de distribuição física de produtos refrigerados, para melhor controle do transporte de produtos refrigerados. Ao final deste estudo em andamento espera-se poder implementar os resultados obtidos no planejamento de rotas eficientes, evitando o ganho acumulado de calor, consequentemente diminuindo a temperatura adquirida pelo produto no decorrer da realização da rota de distribuição dos produtos.

Assim, este artigo está estruturado em quatro etapas: referencial teórico para análise dos estudos de distribuição física de produtos da cadeia do frio; estudo e implementação dos modelos matemáticos estabelecidos por Carvalho (2013) para compreensão do comportamento térmico da carga no decorrer da rota de entrega dos produtos; análise dos resultados da simulação dos modelos e por fim, considerações finais.

\section{REFERENCIAL TEÓRICO}

\section{REFRIGERAÇÃO VEICULAR}

No processo de entrega de produtos da cadeia do frio (resfriados, refrigerados e congelados), existem comportamentos operacionais praticados pelas indústrias e varejos que devem ser seguidos para melhor monitoramento das etapas críticas da cadeia do frio, tais como: a entrega e o recebimento da 
mercadoria com pontualidade, evitando uma exposição desnecessária da carga em ambientes não capacitados para estoque. Também é tratado como comportamento operacional ideal o respeito do colaborador com as normas de manuseio da carga e sistema de refrigeração, tornando todo o processo mais eficiente. Na etapa de carregamento da mercadoria no veículo refrigerado temse que o produto já deve estar com sua temperatura definida anteriormente, pois o modal de transporte não é projetado para resfriá-la, mas sim, para manter a mercadoria dentro dos limites térmicos (Estrada-Flores e Eddy, 2006; Pereira et al., 2010). Sabe-se que em todos os processos onde há fator humano pode haver falhas de operação, sendo muitas vezes o produto armazenado indevidamente, com excessiva espera nos terminais, ocorrendo de maneira lenta o carregamento e descarregamento dos produtos, além da porta de isolamento do veículo se manter aberta excessivamente sem devidos cuidados do operador, entre outras questões (CARVALHO 2013). Assim, há necessidade de aprimoramento nas operações logísticas, portanto o conhecimento das propriedades térmicas envolvidas nestas etapas tem sido foco de estudos recentes.

Para melhor descrever o comportamento das propriedades de refrigeração veicular utilizou-se do modelo matemático estabelecido por Hoang et al. (2012), que considera um modelo físico simplificado dos comportamentos das vazões mássicas de ar dentro de um contêiner refrigerado conforme ilustrado na Figura 1.

Figura 1 - Modelo físico simplificado

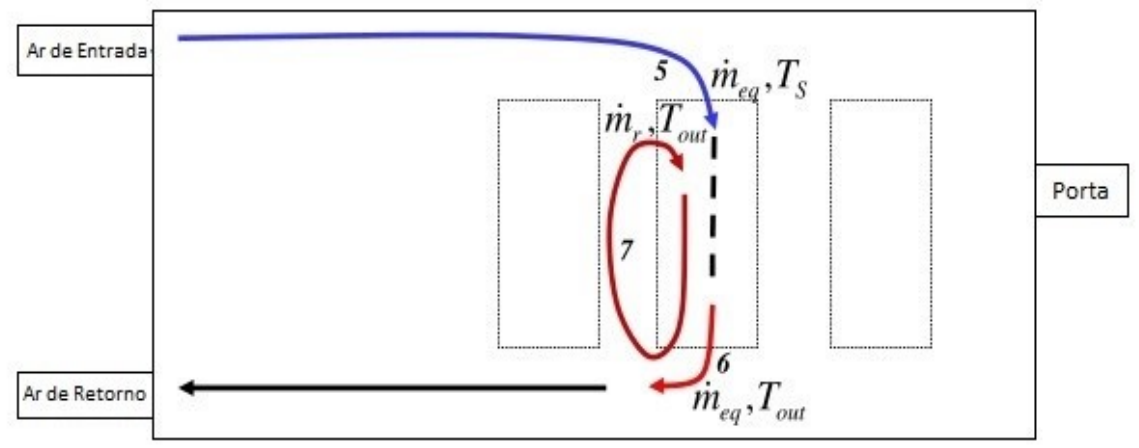

Fonte: Hoang et al. (2012)

Onde (5) representa o fluxo de ar que sai do sistema de refrigeração; (6) fluxo de ar após a troca de calor com a carga, representada pelo retângulo pontilhado; (7) recirculação do ar. Este modelo matemático leva em consideração alguns comportamentos físicos como a troca de calor do ar refrigerado com a carga via convecção, entrada do ar externo como consequência da abertura de portas e ganho de calor através das paredes do sistema (HOANG et al., 2012).

Através da modelagem proposta pelos autores supracitados, Carvalho (2013) adapta o modelo para ser aplicado a um sistema de roteirização dinâmico com possíveis alterações de rota no decorrer das entregas. A capacidade de isolamento do baú do caminhão diminui com o tempo percorrido e a temperatura interna do veículo de cargas não é apenas influenciada pela troca de calor por suas paredes, mas também pela abertura de portas na operação de carga e descarga. Tem-se também que a variabilidade da temperatura da carga se 
correlaciona com o tempo requerido para a unidade de transporte recuperar o controle de temperatura após a abertura de portas, e a diferença entre o valor térmico máximo e mínimo atingido durante a etapa de carga e descarga (ESTRADA-FLORES e EDDY, 2006; HOANG et al., 2012). Portanto, a abordagem de Carvalho (2013) para controle constante da temperatura da rota durante a entrega, tem como objetivo evitar o aumento térmico acumulado na carga, planejando e atualizando a rota ideal em cada processo de carga e descarga.

Os alimentos de maneira geral podem ser considerados de alta sensibilidade, e quando inseridos em ambientes não apropriados sua deterioração ocorre mais rapidamente. Segundo a Food and Agriculture Organization (2013) 54\% do desperdício de alimentos no mundo ocorre na fase inicial da produção, manipulação pós-colheita e armazenagem. Os restantes $46 \%$ ocorrem nas etapas de processamento, distribuição e consumo. Isso se dá principalmente pela falta de controle necessário no transporte e armazenamento das mercadorias. Tal fato justifica o estudo de roteirização para a distribuição física dos produtos da cadeia do frio.

\section{PROBLEMA DE ROTEIRIZAÇÃO DE VEÍCULOS}

O estudo de roteirização tem seu início na tentativa de solucionar problemas encontrados na distribuição física de produtos, os quais atrapalham o planejamento logístico (NOVAES 2007). Ela é definida por Ballou (2006) como sendo uma atividade que tem por objetivo encontrar um ou mais trajetos ótimos que um veículo deve realizar através de uma malha. Segundo Laporte (1992), a roteirização desempenha papel fundamental na logística e distribuição física de produtos e mercadorias comercializadas, pois possibilita, a partir da utilização de modelos matemáticos adotados segundo a especificidade de cada problema, um melhor planejamento e execução de rotas de entregas. De maneira geral o objetivo clássico do problema da roteirização de veículos, conhecido como vehicle routing problem (VRP), é a redução de custos operacionais no sistema de distribuição, e consequentemente, de tempo e distâncias percorridas no trajeto. Outras variáveis podem ser incluídas na modelagem matemática com a finalidade de aproximá-los do problema real, tais como: restrições de horário em certos trechos devido a leis urbanas, horários limites para entregas em certos clientes, entre outros (CARVALHO 2013).

$\mathrm{Na}$ literatura podem-se encontrar diversos modelos existentes de roteirização, entre eles destaca-se o modelo chamado Problema do Caixeiro Viajante (PCV), conhecido também como Traveling Salesman Problems (TSP). O PCV tem como objetivo traçar a menor rota possível no trajeto total, ou seja, a menor distância possível visitando todos os pontos (de entrega) uma única vez (CUNHA, 2000). Pode-se considerar este modelo como sendo básico, pois ignora uma gama de variáveis e restrições encontradas em problemas reais, portanto, otimizações para o modelo são necessárias para a devida utilização em casos mais complexos. Segundo Carvalho (2013), há pesquisas que utilizam PCV otimizados. Exemplos de trabalhos com esta abordagem são: Christofides (1985); Laporte e Nobert (1987); Desrochers, Lenstra e Savelsbergh (1990); entre outros. Nestes modelos são incorporadas variáveis como: janela de tempo, capacidade do veículo, distância máxima da rota, custo máximo, entre outros. Quanto à complexidade computacional, o PCV é um dos poucos problemas que estão 
definidos como "NP-Hard", o que significa que sua ordem de complexidade é exponencial. Em termos práticos, quanto maior for o número de pontos em um PCV, o esforço computacional para resolvê-lo eleva-se exponencialmente. Logo, não há possibilidade de resolver o problema real em tempo hábil, porém, ao aplicarem-se métodos heurísticos pode-se ter um resultado aproximado do ótimo.

Um método heurístico que é utilizado nesta área de estudo é o "Método do Vizinho mais Próximo", que segundo Cunha et al. (2002) considera o seguinte: um veículo de transporte inicia sua trajetória de viagem em um ponto qualquer do grafo e então segue para um ponto que esteja mais próximo; a partir deste, busca-se outro ponto mais próximo ainda não visitado e assim sucessivamente até que todos os pontos sejam visitados, retornando então para o ponto de origem.

O problema de roteirização pode ser classificado em estático ou dinâmico (GHIANI et al. 2007). A roteirização estática é definida por um problema onde todas as variáveis são conhecidas antes do início do processo de roteirização sem mudanças ao longo dos trajetos. De maneira análoga, a roteirização dinâmica se dá por problemas onde as variáveis (temperatura, velocidade, entre outras) podem mudar ao decorrer da rota, portanto, é o método mais próximo de situações reais. Sistemas modelados de maneira dinâmica permitem obtenção de informações em tempo real, consequentemente, oferecem a possibilidade de tomada de decisões em estudos com cenário dinâmico (NOVAES e BURIN, 2010).

O problema de transporte de cargas refrigeradas pode ser modelado como um problema de roteirização dinâmica, por conta do controle necessário da temperatura ao decorrer da rota. Tem-se que as características da rota influenciam diretamente na temperatura interna do veículo, tais como: o número de paradas para realizar as entregas, onde as portas do baú de carga são abertas, fazendo com que haja uma troca de calor entre o ambiente externo com o interno; quantidade de produtos a serem entregues em um ponto; tempo do trajeto entre pontos, etc. Portanto, a qualidade final da carga refrigerada está relacionada à eficiência da rota escolhida.

Figura 2 - Exemplo de rotas alternativas $x$ qualidade dos produtos

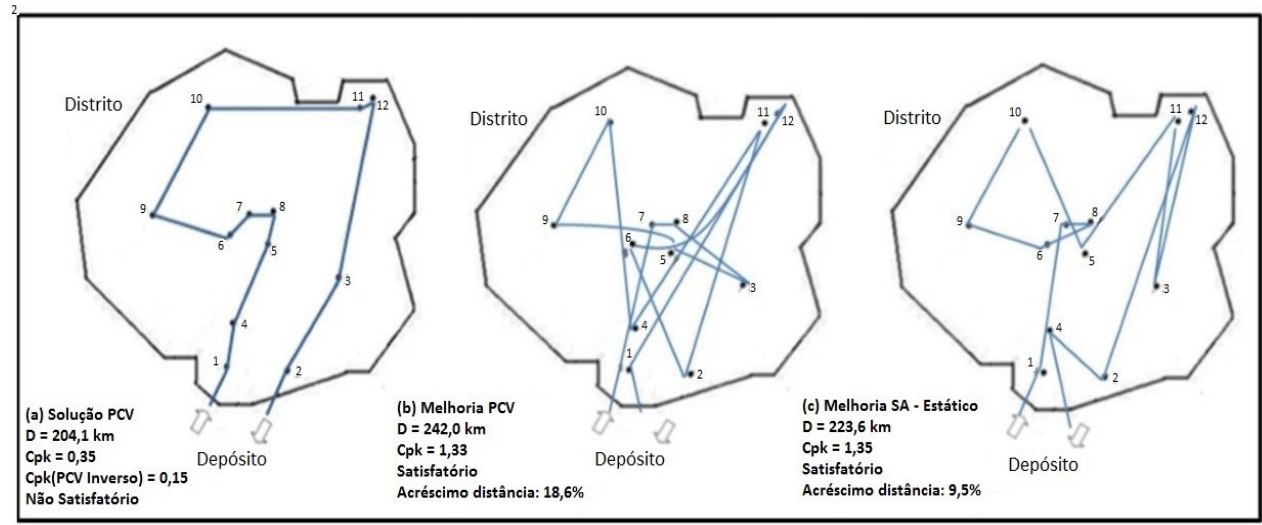

Fonte: Carvalho (2013)

A Figura 2 apresenta um mesmo plano de entregas, porém com 3 rotas diferentes, observando-se que na solução (a), onde foi utilizado o modelo PCV 
com a heurística do vizinho mais próximo, a qualidade da carga foi não satisfatória. Já nos planos de roteirização (b) e (c) a qualidade foi mantida dentro dos padrões estabelecidos pelo autor, com um acréscimo na distância de $18 \%$ e $9,5 \%$ respectivamente. Assim, no caso onde o foco é a temperatura ideal para a carga, comprova-se que quando uma rota não é ótima para o problema tem-se uma perda de qualidade nos produtos. Tem-se que neste estudo o sistema de refrigeração opera enquanto o veículo desloca-se entre os pontos de entrega, portanto, quanto menor for esta distância percorrida, menos o sistema irá resfriar os produtos. Logo, se em uma rota houver muitas entregas com pequenos deslocamentos, a temperatura do produto se aproximará mais rapidamente da temperatura ambiente externa ao veículo devido às paradas e abertura de portas do mesmo.

Carvalho (2013) estudou um caso de roteirização dinâmica onde a variável a ser controlada foi a temperatura do produto. Elaborou-se um modelo matemático, onde a temperatura está em função do tempo, para simulação do processo de entregas (deslocamento e descargas), e através disso possíveis rotas onde a capacidade térmica do produto não é ultrapassada, visando o equilíbrio da qualidade do produto com o custo do trajeto.

\section{MATERIAIS E MÉTODOS}

Esta seção apresenta o estudo do modelo matemático proposto por Carvalho (2013), o qual será utilizado juntamente com o modelo de roteirização a ser desenvolvido para a distribuição de produtos da cadeia do frio.

\section{ROTEIRIZAÇÃO DE VEÍCULO REFRIGERADO JUNTO À OTIMIZAÇ̃̃O LOGÍSTICA}

De acordo com Estrada-Flores e Eddy (2006), tem-se que a temperatura interna de um veículo utilizado para distribuição física de produtos da cadeia do frio está relacionada com a temperatura inicial e final de um processo de entrega durante a abertura de portas e, o tempo de deslocamento de um ponto a outro, pois é neste processo que o sistema de refrigeração opera para manter o controle térmico do veículo de carga. Logo, quanto menor for o tempo de deslocamento entre dois pontos de entrega de produtos, maior será o acúmulo de temperatura durante o processo de entrega. Portanto, ao realizar a modelagem matemática deste problema, objetiva-se impedir que os limites máximos e mínimos de temperatura do produto transportado sejam violados, e assim, verifica-se se a rota estipulada para realizar a distribuição dos produtos pode gerar prejuízos aos produtos quanto ao aumento térmico não esperado e, então se analisam novas rotas. A Figura 3 ilustra dados de tempo e temperatura em uma rota de um caminhão de entrega, onde se pode verificar a relação do acúmulo de temperatura do produto e a relação das temperaturas iniciais e finais do processo de entrega. Com um total de 12 clientes, os números (1 a 12) da Figura 3 indicam o intervalo de tempo de descarga do veículo no respectivo cliente, sendo o cliente número 8 , o que possui o maior intervalo, de aproximadamente 60 minutos. Line-haul I e II é o processo da modelagem de Carvalho (2013) conhecido como Linha de Curso, que será discutido posteriormente. 
Figura 3 - Comportamento técnico do veículo em relação ao roteiro de entrega

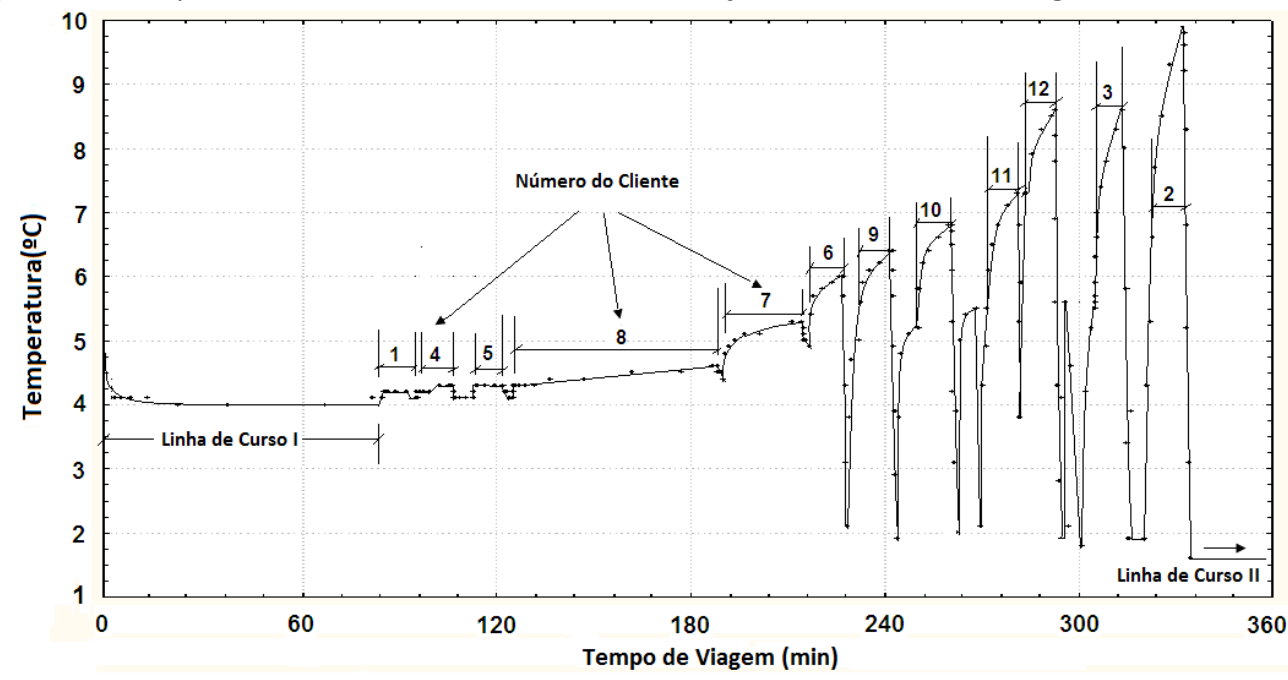

Fonte: Carvalho (2013)

\section{MODELAGEM MATEMÁTICA DE COMPORTAMENTO TÉRMICO DE PRODUTOS}

Ao iniciar o estudo do modelo proposto por Carvalho (2013), deparou-se com três modelos matemáticos termodinâmicos, cada um responsável por uma etapa da rota durante a execução da distribuição de produtos da cadeia do frio.

Para fins de exemplificação, considere um ponto de origem (PO), de onde parte o veículo que fará entregas de produtos refrigerados e, 5 pontos adicionais (P1, P2, P3, P4 e P5), considerados como pontos de entregas.

O veículo parte da origem (PO) desloca-se até o primeiro cliente (P1), realiza a descarga planejada, segue viagem até o ponto subsequente definido pela rota planejada, realiza nova entrega, e assim sucessivamente, até que por fim, entrega toda a carga existente em seu interior, retornando vazio ao ponto de origem (PO) da rota.

Verifique a Figura 4, onde LDC1 e LDC2 são, respectivamente, Linha de Curso 1 e 2 . 
Figura 4 - Estrutura da modelagem de entrega

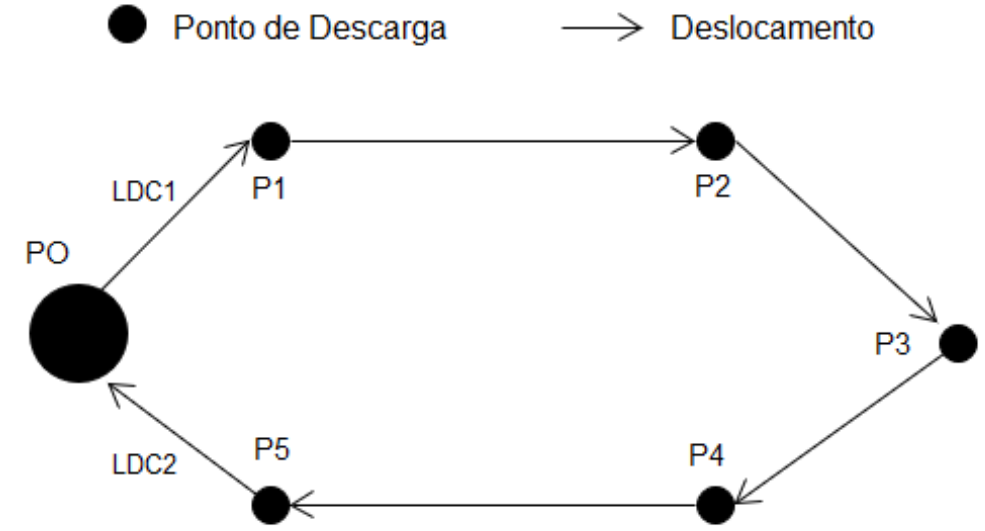

Fonte: Autores, baseado em Carvalho (2013)

Adaptando a modelagem feita por Hoang et al., (2012), Carvalho (2013) apresenta uma equação para o cálculo da temperatura do produto interno ao veículo em função do tempo, considerando-a uniforme:

$$
\theta(\tau)=\theta_{0}+\left(\theta_{\text {load }}-\theta_{0}\right) \cdot \exp \left(\frac{-H_{i} \cdot \tau}{m_{i} \cdot C_{i}}\right)
$$

Onde $\theta(\tau)$ representa a temperatura do produto $i$ no tempo $\tau, \theta_{0}$ refere-se à temperatura inicial do compartimento de carga (ex. um contêiner), $m_{i}$ representa a massa total do produto $(\mathrm{kg}), \theta_{\text {load }}$ representa a temperatura do compartimento após o carregamento do produto $i, H_{i}$ é a condutância do produto $i$ e $C_{i}$ representa a capacidade térmica do produto $i$.

O estudo de caso realizado por Carvalho (2013) procurou simular um problema de entregas de cargas refrigeradas buscando uma rota otimizada, ou seja, buscar manter a qualidade do produto dentro dos limites aceitáveis com o menor custo possível. Os dados de tempo da rota e temperatura do produto foram retirados através de uma simulação realizada no programa CoolVan, que é um software desenvolvido pelo Food Refrigeration and Process Engineering Research Center da Universidade de Bristol, Reino Unido.

"Ele tem como objetivo prever temperaturas durante um roteiro de multi entregas de alimentos refrigerados e congelados, além de ajudar o projeto e operação de veículos de entrega para pequenos e médios roteiros" (GIGIEL et al. 1998; JAMES e SCHOFIELD, 1998; JAMES et al. 2006; MANUAL COOLVAN, 2000; apud CARVALHO 2013).

Como já comentado, na simulação foram estipulados dados de temperatura do produto e tempo de viagem, logo, a equação (1) precisou ser calibrada e adaptada em cada etapa do processo de entrega apresentado na Figura 4, para que seus resultados estejam conforme os dados obtidos através do CoolVan.

A primeira etapa de estudo trata do comportamento das Linhas de Curso I e II, responsáveis por calcular a temperatura interna do veículo desde o ponto de origem (PO) até o primeiro ponto de entrega (P1), LDC1, e do último cliente visitado retornando ao ponto de origem (PO), LDC2. Assim, tem-se a equação: 


$$
\theta(j)=\theta_{j-1} \exp \left(\frac{\beta \cdot d t_{j-1, j}}{m}\right)
$$

Sendo $\theta(j)$ a temperatura do $\operatorname{ar}\left({ }^{\circ} \mathrm{C}\right)$ no ponto $j, \theta_{j-1}$ é a temperatura inicial do produto no momento de saída (uniforme), $m$ é a razão de massa total contida no veículo $(\mathrm{kg}), d t$ é o intervalo de tempo ( $\mathrm{min}), \beta$ é o coeficiente a ser calculado pela calibração dos dados obtidos pela simulação do CoolVan na etapa atual, ou seja, ele é responsável por tornar os resultados dos modelos matemáticos aplicados próximo aos resultados do simulador. Aplicando In em (2) tem-se:

$$
\beta=\frac{\ln \left(\frac{\theta(j)}{\theta_{j-1}}\right)}{\frac{d t_{j-1, j}}{m}}
$$

Assim que a unidade de transporte chega em um ponto de entrega, a etapa de descarga se inicia (neste estudo, considera-se a segunda etapa). Logo, ocorre a abertura das portas do veículo para acesso à carga. Enquanto o operador realiza a função desta etapa há uma troca de calor do ar externo com o interno durante todo o processo, comportamento este expresso pela equação a seguir:

Equação de Descarga:

$$
\theta(\tau)=\alpha_{1} \cdot \theta_{0} \cdot \theta_{\text {ext }}^{\alpha_{2}} \cdot \exp \left(\beta_{\text {unl }} \cdot \frac{\tau}{Q_{i}}\right)
$$

Sendo $\tau$ o tempo em minutos, $\theta_{\text {ext }}$ a temperatura externa ao ambiente, temse que esta temperatura não muda significativamente no decorrer do processo de entrega, logo, faz-se uma média do valor medido no início com o final da etapa. Os coeficientes $\alpha_{1}, \alpha$ e $\beta_{\text {unl }}$ foram ajustados através da regressão dos dados adquiridos por Carvalho (2013) com o simulador CoolVan (verificar Tabela 1). O valor de $Q_{i}$ é constante, sendo uma fração da carga total obtido através da média aritmética das frações de carga inicial e final do processo de entrega.

Tabela 1 - Dados dos coeficientes do modelo de entrega

$\begin{array}{cc}\text { Coeficientes } & \text { Valor } \\ \alpha_{1} & 0.3234 \\ \alpha_{2} & 0.4517 \\ \beta_{u n l} & 0.00081\end{array}$

Fonte: Adaptado de Carvalho (2013)

A terceira e última fase do processo é o deslocamento entre os pontos de entrega da rota, o qual tenta ajustar a temperatura para o limite médio definido; ou seja, quanto mais tempo a unidade de transporte percorrer entre os pontos de entrega, mais próxima do limite de temperatura inferior a carga estará. A equação (5) representa o modelo matemático que simula esta etapa do processo.

Equação de Deslocamento:

$$
\theta_{t v l}=\alpha_{1} \cdot \theta_{0} \cdot \tau^{\alpha_{2}} \cdot Q_{i B}^{\alpha_{3}}
$$

Sendo $\theta_{t v l}$ a temperatura do veículo quando ele chega ao próximo cliente, $\tau$ é o tempo do trecho entre o cliente $i$ e $i+1, Q_{i}$ é a razão de massa total dentro do veículo; $\alpha_{1}, \alpha_{2}$ e $\alpha_{3}$ são os coeficientes que foram ajustados para estarem próximos dos resultados térmicos obtidos pelo simulador, os quais se encontram na Tabela 2. 
Tabela 2 - Dados dos coeficientes do modelo de deslocamento

\begin{tabular}{cc}
$\alpha_{1}$ & Valor \\
$\alpha_{2}$ & 1.094 \\
$\alpha_{3}$ & -0.07446 \\
\hline & 0.10159
\end{tabular}

Fonte: Adaptado de Carvalho (2013)

\section{MODELAGEM MATEMÁTICA DA DISTRIBUIÇÃO DE PRODUTOS DA CADEIA DO FRIO}

Após o estudo e compreensão dos modelos matemáticos propostos, os mesmos foram implementados através da simulação para analisar e avaliar os resultados obtidos, no intuito de utilizá-los para possíveis aplicações reais. Para realização desta etapa foram estabelecidos cinco pontos de entrega (aqui considerados como clientes, numerados de 2 a 6) contendo dados de tempo de carga e de descarga (o tempo que a porta do veículo fica aberta), uma matriz de tempo de viagem entre um ponto $i$ (cliente) para todos os outros pontos (considerando o ponto de origem como sendo o ponto 1 ) e a razão de massa entregue nestes pontos. Os dados podem ser verificados na Tabela 3, juntamente com a matriz de tempo, na Tabela 4.

Tabela 3 - Dados utilizados na simulação

\begin{tabular}{ccc} 
Pontos & $\begin{array}{c}\text { Tempo de Descarga } \\
(\mathrm{min})\end{array}$ & Razão de Massa $(\%)$ \\
2 & 20,0 & 15 \\
3 & 35,0 & 30 \\
4 & 20,0 & 15 \\
5 & 10,0 & 10 \\
6 & 25,0 & 30 \\
\hline
\end{tabular}

Fonte: Autores (2018)

Tabela 4 - Matriz de tempo entre pontos, Origem/Destino (min)

\begin{tabular}{ccccccc} 
O/D & 1 & 2 & 3 & 4 & 5 & 6 \\
$\mathbf{2}$ & 0 & 8.4 & 10.2 & 15 & 18 & 16 \\
3 & 8.4 & 0 & 4.8 & 10 & 15 & 29 \\
$\mathbf{4}$ & 10.2 & 4.8 & 0 & 6.1 & 3.9 & 32 \\
$\mathbf{5}$ & 15 & 10 & 6.1 & 0 & 4.3 & 27 \\
$\mathbf{6}$ & 18 & 15 & 3.9 & 4.3 & 0 & 24 \\
\hline
\end{tabular}

Fonte: Autores (2018)

Neste caso, na matriz de tempos, tem-se que o valor de $a_{i j}$ representa o tempo (em minutos) que o veículo leva para percorrer entre o ponto $i$ até o ponto $j$. 
Utilizando-se do programa $\operatorname{MATLAB}^{\circledR}$ (R2013a) foi possível desenvolver um algoritmo que realiza todas as etapas do processo de entrega, gerando dados para análise dos resultados obtidos.

\section{MODELAGEM MATEMÁTICA DOS CUSTOS DE REFRIGERAÇÃO X ROTAS}

Para ter-se uma análise quantitativa dos valores de estudo de roteirização, foram estabelecidos valores de consumo de combustível conforme distância e tempo percorridos entre os pontos de entrega. Alguns parâmetros foram adotados para permitir um melhor estudo integrado do sistema de refrigeração do veículo durante a realização da distribuição física dos produtos da cadeia do frio, tais como: o sistema de refrigeração é responsável por cerca de $20 \%$ do consumo total (dado definido pelos autores para continuidade das análises); e durante o processo de descarga o veículo juntamente com seus equipamentos estarão desligados. Para estudo inicial, considerou-se a velocidade do veículo como constante de $(50 \mathrm{~km} / \mathrm{h})$ em todo o processo de entrega.

Para o cálculo do custo total do trajeto, considerou-se a seguinte equação:

$$
\text { P.C.V. } \tau=\$
$$

Onde $P$ é o preço do combustível ( $3 \$ /$ litro), $C$ é o consumo do veículo $(0,2$ litro $/ \mathrm{km}), V$ é a velocidade média do veículo $(0,83 \mathrm{~km} / \mathrm{min}), \tau$ é o tempo do deslocamento total em minutos e $\$$ é o custo total em unidades monetárias. Os valores dessas constantes foram definidos pelos autores para fins de análise.

Primeiramente, através de uma matriz origem destino entre pontos, foi aplicada a heurística do vizinho mais próximo (Moresco e Silva, 2017) para resolver o PCV (Problema Caixeiro Viajante) no cenário estudado, obtendo-se uma noção do menor custo possível em relação à distância. A construção da rota por este método inicia pela origem (centro de distribuição), e o próximo ponto a ser visitado sempre será o que estiver mais próximo do ponto atual (ver Figura 5), processo que irá ocorrer até todos os pontos da rota terem sido visitados uma única vez, sendo o destino final a origem.

A heurística do Vizinho Mais Próximo pode ser resumida nos seguintes passos:

a) Escolha um ponto de entrega como ponto atual.

b) Procure o trecho de menor peso (pode ser dado em distância) que liga o ponto atual a um ponto não visitado $P$.

c) Faça o ponto atual ser $P$.

d) Marque P como visitado.

e) Quando todos os pontos forem visitados, a heurística se encerra.

f) Caso contrário, volte para o passo b. 
Após o trajeto ser definido através do problema de roteirização estudouse o comportamento térmico da rota mediante simulação dos modelos matemáticos propostos e analisaram-se os custos obtidos. Assim, para este sistema (roteirização com a análise térmica da rota gerada), buscou-se estabelecer um algoritmo, denominado "Controle", capaz de controlar a temperatura do produto transportado mediante mudanças do primeiro trajeto encontrado pelo modelo de roteirização. Caso a temperatura, em algum ponto $i$, exceda um limite estabelecido, o algoritmo irá buscar rotas alternativas a partir de $i-1$ com base na primeira rota gerada.

Figura 5 - Explicação da heurística do vizinho mais próximo

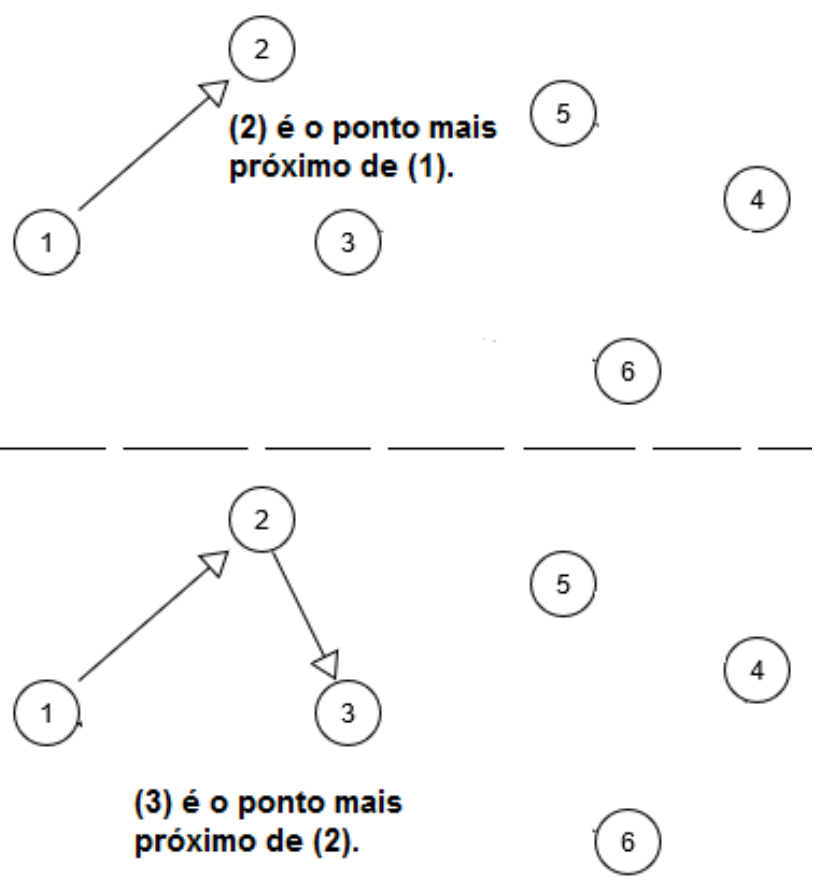

Fonte: Autores (2018)

\section{RESULTADOS E DISCUSSÃO}

Após o estudo dos modelos matemáticos propostos por Carvalho (2013) sobre a performance térmica durante a realização de uma rota de entrega de produtos da cadeia do frio, os mesmos foram implementados em um modelo de simulação no intuito de avaliar os resultados obtidos quanto ao tempo de viagem entre os pontos de entrega e, temperatura do produto transportado.

Os valores contidos no Gráfico 1 foram gerados pela simulação através de dados pré-definidos, tendo como rota os pontos $1 \rightarrow 2 \rightarrow 3 \rightarrow 4 \rightarrow 5$, podendo perceber uma quebra de continuidade do resultado das temperaturas (obtidas pela implementação das equações) na transição da etapa de descarga com o processo de deslocamento e vice-versa. 
Esse fenômeno de quebra de continuidade ocorre quando as equações de deslocamento e descarga não respeitam as condições necessárias de continuidade, neste caso, quando o tempo da equação for $\tau=0$, a temperatura do modelo deve igualar-se a temperatura inicial fornecida, $\theta(0)=\theta_{0}$.

Gráfico 1 - Comportamento da variação de temperatura x tempo (modelo de Carvalho, 2013)

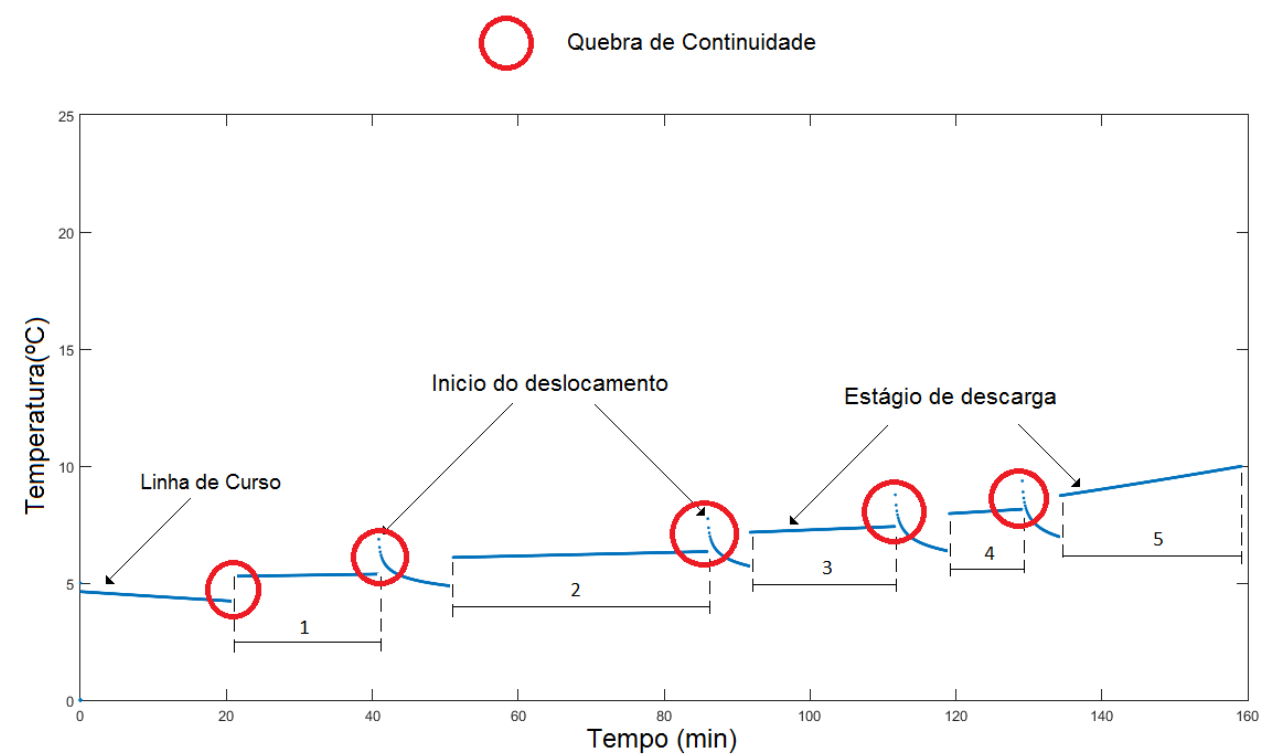

Fonte: Autores (2018)

Buscando melhor compreender o porquê da descontinuidade das equações, estudou-se o comportamento matemático de cada uma delas no intuito de identificar onde a regra de continuidade das equações não são respeitadas. Vale destacar que não se analisou a equação empregada para as linhas de curso, pois não houve quebra de continuidade em sua aplicação.

\section{ANÁLISE DA EQUAÇÃO DE DESCARGA}

Considerando a equação (4) e aplicando $\tau=0$ para verificar a condição de continuidade onde $\theta(0)=\theta_{0}$ tem-se:

$$
\theta(\tau)=\alpha_{1} \cdot \theta_{0} \cdot \theta_{\text {ext }}^{\alpha_{2}}
$$

Deve-se esperar que a temperatura do modelo $\theta(j)$ seja igual à temperatura inicial $\left(\theta_{0}\right)$. Percebe-se que para tal condição ser verdadeira os valores que multiplicam $\theta_{0}$ devem ser igual a 1 , ou seja, $\alpha_{1} \cdot \theta_{\text {ext }}^{\alpha_{2}}=1$, situação que não condiz com a realidade do modelo, pois os valores de $\alpha_{1}$ e $\theta_{\text {ext }}$ são constantes no estudo e diferentes de 1 . Portanto, pode-se afirmar que o modelo de descarga, apresentado por Carvalho (2013) não respeita a condição de continuidade, responsável por manter as temperaturas contínuas no decorrer da simulação. Se esta condição for violada têm-se valores de temperatura que resultam em saltos, um comportamento não real em situações normais de distribuição de produtos. 


\section{ANÁLISE DA EQUAÇÃO DE DESLOCAMENTO}

Da mesma forma que no estudo anterior, aplicando $\tau=0$ na equação (5) encontra-se:

$$
\theta_{t v l}=0
$$

Portanto, pode-se perceber que há uma quebra na continuidade da equação de deslocamento por conta do resultado obtido na equação (8), pois em toda e qualquer temperatura inicial $\theta_{0}$ dada, quando o tempo for $\tau=0$, ter-se-á obtido o resultado $\theta_{t v l}=0$, valor não condizente com a realidade, pois no tempo nulo a temperatura deve estar inalterada. Assim, o valor esperado para o modelo nestas condições está apresentado na equação (9), representando a temperatura inicial do sistema, pois se o tempo é nulo, significa que ainda não houve alterações na temperatura do produto.

$$
\theta_{t v l}=\theta_{0}
$$

\section{PROPOSTA DE UM MODELO MATEMÁTICO PARA ANÁLISE DA PERFORMANCE TÉRMICA DA CARGA}

Por conta do problema de quebra de continuidade analisado anteriormente, e como forma de contribuição para o estado da arte sobre este tema de estudo, propôs-se para este trabalho um novo modelo para substituir as equações (4) e (5); buscando manter a continuidade do comportamento térmico da carga durante todo o processo de descarga e deslocamento:

$$
\begin{gathered}
\tau \equiv \text { Tempo (min) } \\
\theta_{0} \equiv \text { Temperatura inicial }\left({ }^{\circ} \mathrm{C}\right) \\
m \equiv \text { Massa no caminhão (\%) } \\
\beta \equiv \text { Coeficiente térmico do produto } \\
\theta(\tau) \equiv \text { Temperatura no tempo } \tau\left({ }^{\circ} \mathrm{C}\right) \\
\theta_{\text {fonte }} \equiv \text { Temperatura do ambiente externo }\left({ }^{\circ} \mathrm{C}\right) \\
\theta(\tau)=\theta_{\text {fonte }}+\left(\theta_{0}-\theta_{\text {fonte }}\right) \cdot \exp \left(\beta \cdot \frac{\tau}{m}\right)
\end{gathered}
$$

Onde $\theta(\tau)$ representa a temperatura $\left({ }^{\circ} \mathrm{C}\right)$ do produto no tempo $\tau(\min )$, $\theta_{0}$ é a temperatura inicial do produto, $m$ é a razão de massa de produto contida no caminhão, $\beta$ é o coeficiente de variação de temperatura, o qual será discutido adiante, e $\theta_{\text {fonte }}$ representa a temperatura que o sistema exerce sobre o produto, assumindo os valores $4^{\circ} \mathrm{C}$ quando no estado de deslocamento entre pontos e $20^{\circ} \mathrm{C}$ quando está sendo realizada uma entrega. Tais valores foram definidos pelos autores para estudo do comportamento do modelo. Substituindo 
as equações (4) e (5) pela equação (10), tem-se um novo comportamento para a variação de temperatura no produto (ver Gráfico 2).

Gráfico 2 - Comportamento da variação de temperatura $x$ tempo (modelo proposto)

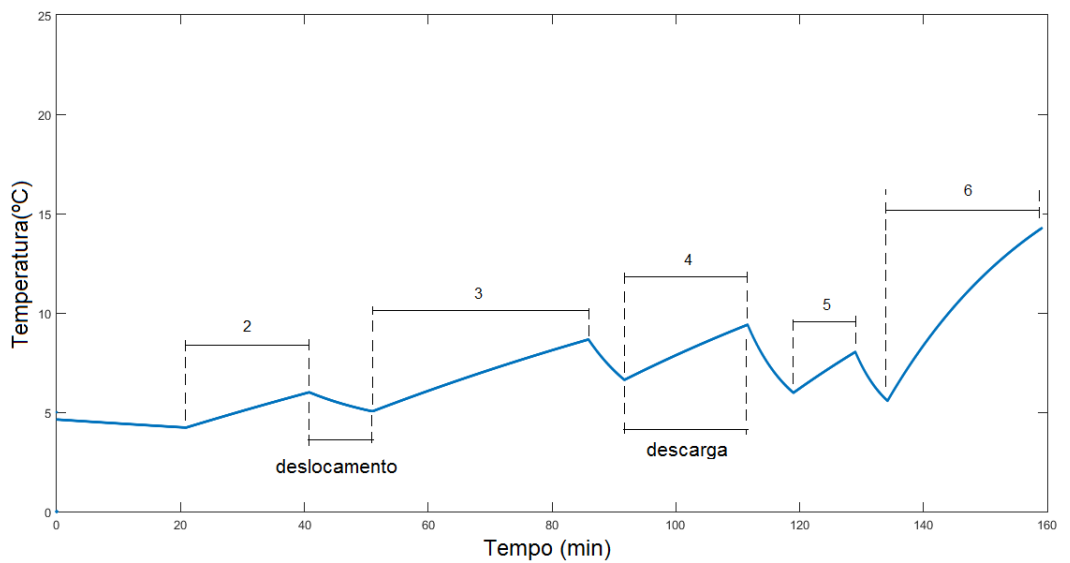

Fonte: Autores (2018)

Nestas condições, tem-se um comportamento contínuo em todo o percurso de entrega, onde os trechos com aumento de temperatura representam os momentos em que ocorre a descarga do produto em determinado ponto/cliente; já onde há declínio da temperatura ocorre o deslocamento do veículo entre pontos de entrega de produtos. A taxa de crescimento ou decrescimento térmico da carga é dada pelo coeficiente $\beta$ do modelo, que representa o comportamento da temperatura do produto com o ambiente, e assim, quanto menor o seu valor mais lentamente será o ganho ou perda de temperatura e vice-versa.

\section{ANÁLISE DA ROTEIRIZACCÃO DO VEÍCULO}

Definidas as equações matemáticas que podem ser consideradas contínuas, pode-se realizar a análise térmica da carga refrigerada no decorrer de uma rota de distribuição física. Como relatado na metodologia, as rotas (neste estudo) são definidas mediante a heurística do vizinho mais próximo para resolução do problema de roteirização. Após a obtenção de uma rota, inicia-se um processo de simulação, através do qual se realiza uma análise da temperatura do produto no decorrer do tempo e os custos do trajeto, como combustível e tempo, obtidos durante a realização da rota de distribuição da carga.

\section{Algoritmo de Controle}

Após a implementação do modelo matemático proposto, obteve-se como primeiro resultado da análise, o Gráfico 2. Na sequência, desenvolveu-se o algoritmo "Controle", capaz de buscar uma possível rota que mantenha a temperatura do produto abaixo do limite térmico estabelecido. Ver Figura 6, 
onde se pode perceber que o processo da heurística de roteirização (para o PCV) e a Simulação são parte de sua estrutura (Linhas 4, 5, 18, 20).

Quando implementada a rotina de roteirização, analisa-se uma rota de custo reduzido, obtida através da matriz de tempos enviada como entrada. $A$ rotina de roteirização retorna quatro $(4)$ vetores $(D, V, T, P)$, organizados de acordo com a sequência definida como caminho, sendo eles: um vetor $D$ com as distâncias (o tempo de deslocamento entre pontos), um vetor $V$ contendo os volumes de produto a serem entregues nos pontos de distribuição, $T$ é um vetor do tempo de descarga em cada ponto e $P$ é um vetor que armazena a sequência dos pontos a serem visitados (o caminho).

Figura 6 - Algoritmo de Controle

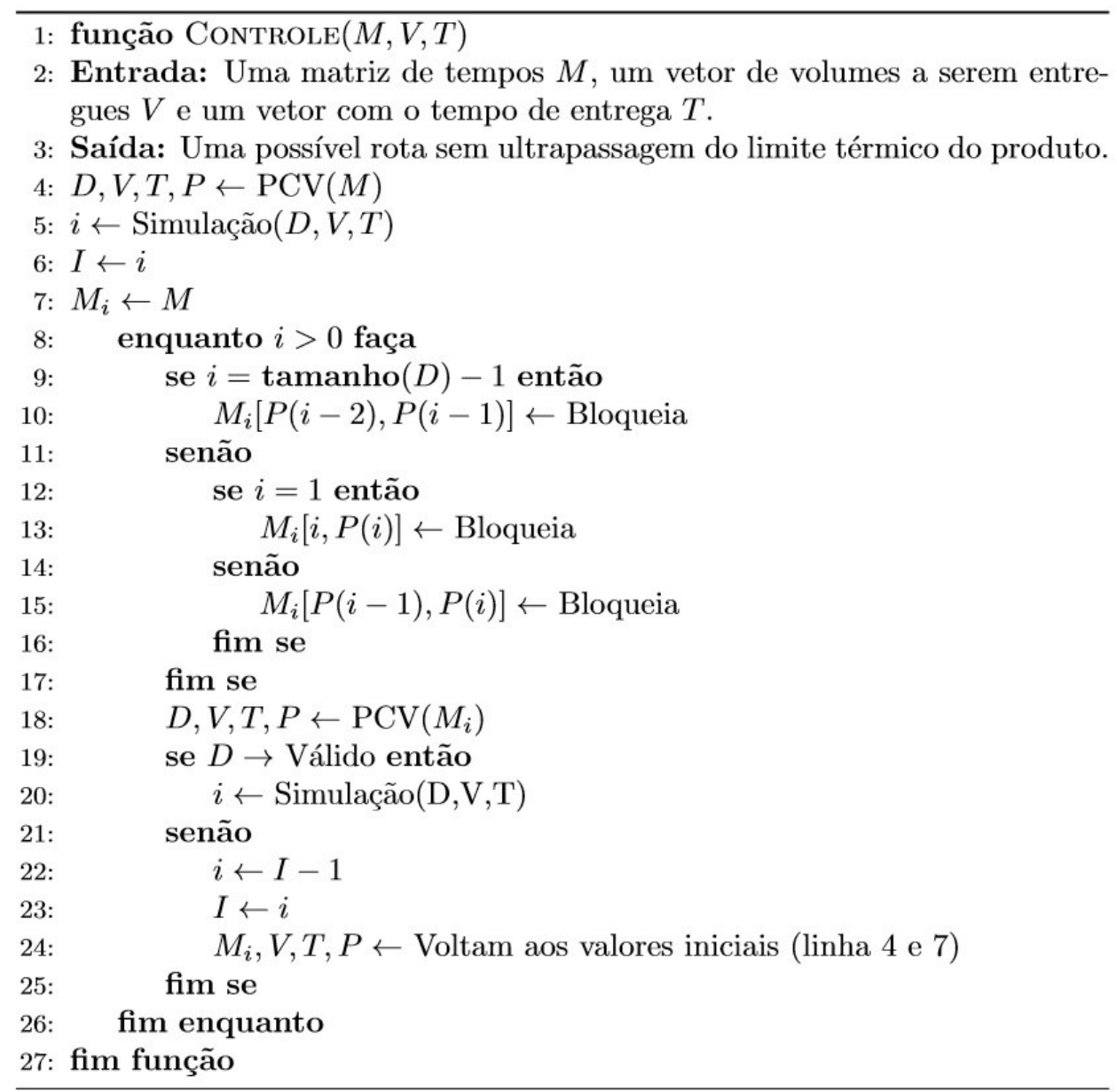

Fonte: Autores (2018)

Após a obtenção da rota, o processo de Simulação é utilizado, o qual recebe como entrada os vetores $D, V$ e $T$, e retorna a posição no vértice $P$, indicando um ponto da rota onde houve um abuso de temperatura (entende-se como abuso de temperatura o fato do produto transportado atingir temperatura acima do limite estabelecido para garantir sua qualidade). Se não houver abuso, $I$ é definido como -1. Ao definir o ponto de abuso de restrição térmica, o algoritmo irá buscar rotas alternativas a partir dos pontos anteriores ao abuso, tornando o caminho de $i-1$ até $i$ inválido na matriz $M_{i}$ e então, aplica-se a rotina de roteirização novamente. Desta forma, obriga-se a realização da busca de um 
caminho diferente, logo, se for possível definir um vetor $D$ o processo de Simulação poderá ser empregado, o qual irá verificar novamente se há abuso do limite térmico. Caso D seja inválido, ou seja, não há solução para o problema de roteirização da matriz $M_{i}$, retorna todos os vetores e a matriz para seu estado inicial e aplica-se $i=I-1$, onde $I$ é o iterador maior. $O$ algoritmo se encerra quando nenhum abuso é identificado ou quando $I \leq 0$, que significa que o sistema de entregas é sem solução, portanto, todas as rotas possíveis foram verificadas e nenhuma respeitou o limite de temperatura.

A informação menos trivial de ser compreendida é o valor de $I$ e o que ele representa. Ele pode ser caracterizado como um iterador que controla as trocas de rotas. É válido comentar que os valores dos pontos de abuso de temperatura (PA) são diferentes dos pontos dos caminhos (PC). Se um abuso ocorrer no ponto " 5 ", significa que na posição 5 do vetor de caminhos ocorreu um abuso de temperatura, ou seja, o ponto de caminho que se encontrar nessa posição foi responsável pelo abuso.

Portanto, $I$ representa uma indicação para PC (ponto de onde novas rotas serão revistas), cujo o valor de $i$ aponta para a posição no vetor de caminho onde se encontrará o PC, e é a partir dali que um novo caminho válido será gerado. Se nenhum caminho válido é gerado, $I$ é decrementado $(I-1)$ e o algoritmo continuado (outra iteração é iniciada).

\section{Análise dos Resultados da Roteirização x Algoritmo}

Para fins de simulação e análise dos resultados do modelo proposto, utilizouse os dados das Tabelas 3 e 4, e o algoritmo resultou em 4 iterações, analisandose 5 rotas e encontrando um possível trajeto sem o abuso de temperatura. 0 limite de temperatura máxima foi estabelecido como $10^{\circ} \mathrm{C}$ para fins de análise.

Tabela 5 - Resultados das Iterações do Algoritmo

\begin{tabular}{cccc} 
Rota & Custo $(\mathrm{R} \$)$ & Temp. Final $\left({ }^{\circ} \mathrm{C}\right)$ & Caminho \\
1 & 21.0 & 10.4 & 23546 \\
2 & 30.7 & 11.8 & 23564 \\
3 & 22.1 & 10.2 & 23456 \\
4 & 33.4 & 10.3 & 23465 \\
5 & 26.1 & 9.8 & 24536 \\
\hline
\end{tabular}

Fonte: Autores (2018)

A primeira análise do algoritmo resultou na rota 1 da Figura 7 (2 3554 6), com custo total calculado pela equação (6) de $\$ 21$ (sendo $\$ 4,2$ o custo representado pelo sistema de refrigeração, que é responsável por $20 \%$ do consumo de combustível do veículo) e o abuso de temperatura ocorre na entrega no ponto 6 , alcançando $10.42^{\circ} \mathrm{C}$. A justificativa para este abuso consiste na forma que a temperatura é acumulada durante o processo de entrega, e assim, o produto é entregue de maneira ineficiente, tendo em vista a temperatura como variável de maior importância. Portanto, na descarga do último ponto o abuso é uma consequência das etapas anteriores. 


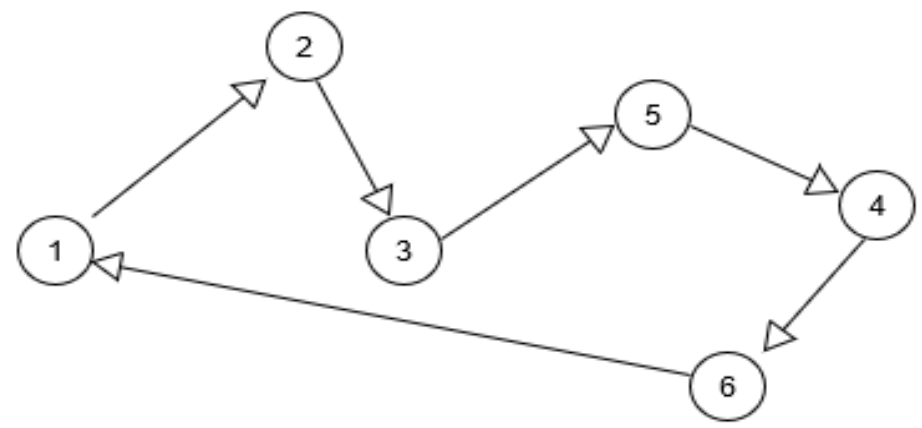

Fonte: Autores (2018)

Figura 8 - Resultados da Rota 1
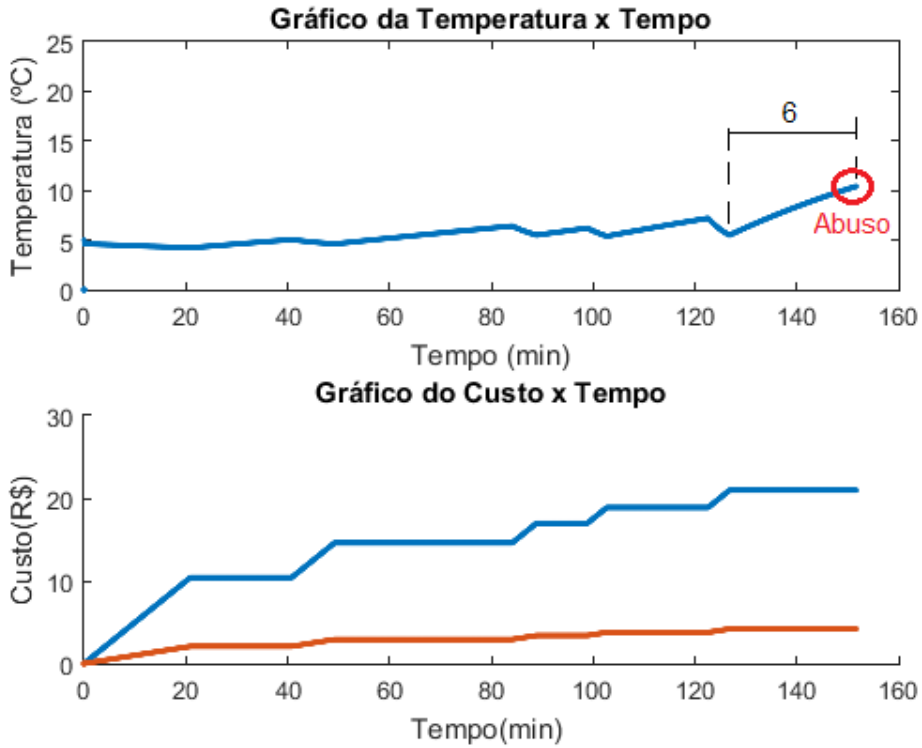

Fonte: Autores (2018)

A Figura 8 representa os resultados obtidos através da análise térmica da rota 1 , sendo que o gráfico superior apresenta o comportamento da temperatura ao decorrer do tempo e o inferior apresenta as curvas de custos, sendo a superior referente ao custo total de transporte e a inferior, referente ao custo da refrigeração.

O resultado final do algoritmo (rota 6) teve um custo total de $\$ 26$ (com $\$ 5,23$ através do sistema de refrigeração) alcançando uma temperatura final de $9.89^{\circ} \mathrm{C}$, que está abaixo do limite estabelecido $\left(10^{\circ} \mathrm{C}\right)$, logo, demonstra que um resultado satisfatório foi atingido. A rota obtida é 24536 , apresentada na Figura 9 e, seus dados de custo e temperatura são apresentados na Figura 10. Da mesma forma como foi analisado o resultado da rota 1 , pode-se estender a explicação para o resultado desta rota 6 . Durante o processo de entrega é comum acontecerem os acúmulos de temperatura, de tal forma, que caso este processo seja realizado de forma ineficiente, em algum ponto a temperatura do 
produto irá ultrapassar o limite estabelecido. A referida rota permitiu encontrar uma sequência de pontos de entrega que diminuiu o acúmulo ganho, fazendo com que a distância de deslocamento entre alguns pontos fosse aumentada, e desta forma, o sistema de refrigeração pôde trabalhar por mais tempo mantendo o controle da temperatura.

Figura 9 - Rota 6, Sem Abuso Térmico

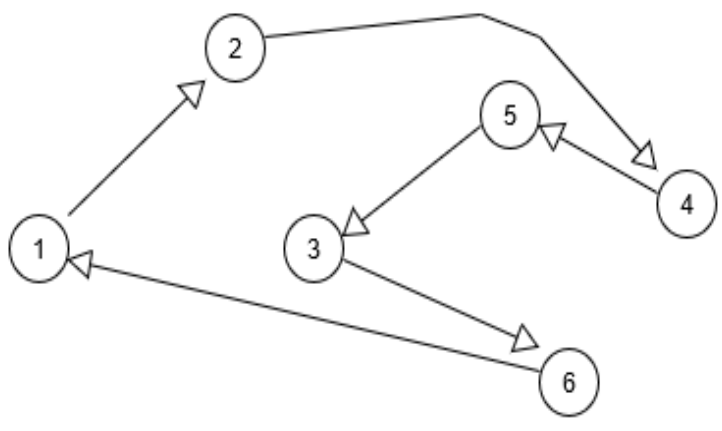

Fonte: Autores (2018)

Vale relembrar que o sistema de refrigeração se mantém desligado durante o processo de entrega, ou seja, os valores de custo tornam-se constantes no mesmo intervalo de tempo onde ocorrer uma entrega.

Pode-se dizer que o algoritmo cumpre com sua finalidade de simular diferentes rotas para a distribuição de produtos considerando a variação de temperatura e assim, um caminho sem abuso de temperatura foi encontrado, porém gerando um acréscimo de aproximadamente $24 \%$ (de \$21 para \$26) no custo total do transporte, quando comparado à rota 6 . 0 aumento no custo ocorre por conta de se escolher trajetos mais longos entre pontos, gerando, consequentemente, um maior consumo de combustível do veículo.

Figura 10 - Resultados da Rota 6
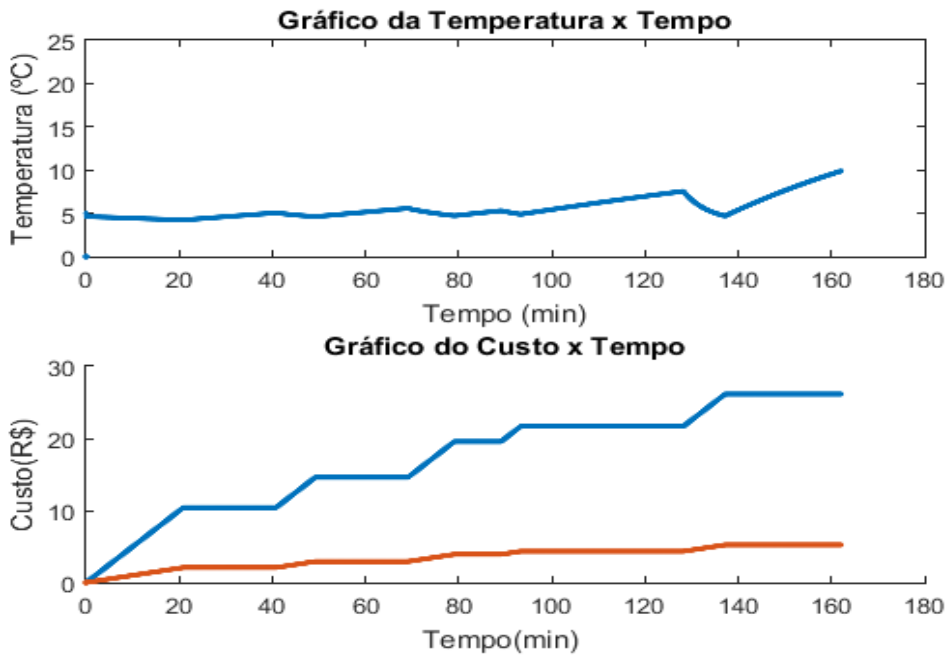

Fonte: Autores (2018) 


\section{CONSIDERAÇÕES FINAIS}

O presente estudo consistiu em analisar artigos e periódicos com ênfase no problema de roteirização e à cadeia do frio, e através disso buscar integrar as duas temáticas de forma que o problema de roteirização pudesse ser considerado dinâmico, ou seja, tendo a temperatura do produto a ser transportado, controlada.

Assim, apresentou-se uma visão geral dos dois temas, e propôs-se um modelo matemático no intuito de encontrar rotas de distribuição de produtos sem a extrapolação da temperatura dos mesmos durante este processo. Para tal objetivo, estabeleceram-se modelos matemáticos capazes de simular a variação de temperatura dos produtos em função do tempo e, também, uma heurística para resolução do problema. Com a utilização destes dois modelos (simulação térmica e roteirização) implementou-se um algoritmo que pôde encontrar um resultado satisfatório. Nem todos os problemas de roteirização de cargas refrigerada terão soluções satisfatórias, pois dependendo do limite térmico e dos dados do sistema estabelecido, pode ser impossível encontrar uma rota onde o produto consiga ser mantido nas temperaturas ideais. A solução para o problema do abuso de temperatura na prática consiste em prolongar as distâncias entre pontos, e desta forma, o sistema de refrigeração pode operar na temperatura do produto durante um intervalo maior de tempo. Portanto, não há nenhuma alteração nos processos de entrega em si, já que o tempo de entrega continuará o mesmo após a aplicação do algoritmo.

Ao produto que requer controle térmico se mantido constantemente abaixo de sua temperatura limite durante o transporte resulta-se na redução de perdas e também no ganho de qualidade. Com o estudo realizado é perceptível que a temperatura do interior do veículo e do produto é fortemente dependente da capacitação dos colaboradores no quesito de distribuição física dos produtos, pois quando há uma baixa qualidade nesse quesito pode-se gerar más práticas de operação, isto é, tempo indevido de portas abertas do veículo, descarregamentos não eficientes, etc. De maneira geral, o resultado obtido neste estudo incipiente não leva em consideração fatores humanos, como atrasos e más operações, porém isto não torna a pesquisa inválida, uma vez que os imprevistos citados acima podem ser incrementados futuramente para aprimoramento do processo, aproximando o estudo da realidade.

Para futuros ajustes e estudos pode ser viável uma análise mais detalhada dos coeficientes do modelo matemático proposto pelos autores, com validações práticas e acréscimos de variáveis no sistema de deslocamento e entrega, como trânsito lento, atrasos humanos e instalações de cortinas de ar, por exemplo. 


\title{
Mathematical modeling for the physical distribution of cold chain products
}

\begin{abstract}
In order to preserve the quality of products that require temperature monitoring some methods and studies are developed on Cold Chain, which is responsible for the logistic chain of the product, from its production, storage to physical distribution to the final consumer. In this context, this article seeks to understand the behavior of the temperature variation of refrigerated loads during its physical distribution and for this, through mathematical modeling, equations are implemented that represent the behavior of thermal variation during the delivery of products, considering the loading processes, displacement and unloading of the same. The objective of this study is therefore to study this behavior in order to generate a simulation tool that is capable of planning routes of physical distribution of refrigerated products, making the whole logistic process more efficient, reducing the undesired internal temperature gain in the vehicle and on the product.
\end{abstract}

KEY-WORDS: Cold chain. Mathematical modeling. Routing. Cooling. Distribution. 
Ballou, Ronald H. Gerenciamento na Cadeia de Suprimentos: planejamento, organização e logística empresarial. 4. Ed. Porto Alegre: Bookmam, 2006

Carvalho, Carolina Correa de. Otimização Dinâmica da Logística de Distribuição de Produtos Alimentícios Refrigerados e Congelados. 2013. 254 f. Tese (Doutorado) - Curso de Faculdade de Engenharia Civil, Arquitetura e Urbanismo, Universidade Estadual de Campinas, Campinas, 2013.

Christofides, N. (1985) "Vehicle routing", in: E.L. Lawler, J.K. Lenstra, A.H.G. Rinnooy Kan and D.B. Shmoys, (eds.). The Traveling Salesman Problem. A Guided Tour of Combinatorial Optimization, Wiley, Chichester, 431-448.

Cunha, C. B. (2000) Aspectos práticos da aplicação de modelos de roteirização de veículos a problemas reais. Transportes, v. 8, n. 2, p. 51. crossref

Cunha, Claudio \& Bonasser, Ulisses \& Abrahão, Fernando. (2002). Experimentos computacionais com heurísticas de melhorias para o problema do caixeiro viajante.

Desrochers, M., Lenstra, J.K., and Savelsbergh, M.W.P. (1990), "A classification scheme for vehicle routing and scheduling problems", European Journal of Operational Research 46, 322-332. crossref

Estrada-Flores, S., Eddy, A. (2006) Thermal Performance Indicators for Refrigerated Road Vehicles, International Journal of Refrigeration, v. 29, pp. 889898. crossref

Food and Agriculture Organization (Roma) (Org.). Desperdício de alimentos tem consequências no clima, na água, na terra e na biodiversidade. 2013. Disponível em: <http://www.fao.org.br/daccatb.asp>. Acesso em: 15 maio 2018.

Ghiani, G., Quaranta, A., Triki C. (2007) New policies for the dynamic traveling salesman problem. Optimization Methods and Software, vol. 22, No. 6, December, 971-983. crossref

Heap, R. D. (2006) Cold chain performance issues now and in the future. IIR Bulletin, n. 4, p. 1-11.

Heap, R.; Kierstan, M.; Ford, G. (1998), Food transportation. Londres: Blackie Academic \& Professional. 
Hoffman, W. (2006). Hot Market, Cool Freight. Journal of Commerce.

http://dx.doi.org/10.1016/j.jfoodeng.2012.06.020.International Institute of Refrigeration - IIR (2004). Temperature indicators and time-temperature integrators: 3rd informatory note on refrigeration and food. Paris.

Isabela Neto Piccirillo; Daiane Maria de Genaro Chiroli; Luciana Torres Correia de Mello. Roteirização com o método da varredura: uma proposta para melhorar a formatação de cargas, reduzir custos e satisfazer cliente. Caracas: Espacios, 2016.

Laporte, G, Louveaux, F. Mercure, H. (1992) The vehicle routing problem with stochastic travel times, Transportation Science 26 (3) 161-170. crossref

Laporte, G., and Nobert, Y. (1987), "Exact algorithms for the vehicle routing problem", in: S. Martello, G. Laporte, M. Minoux and C. Ribeiro (eds.), Surveys in Combinatorial Optimization, North-Holland, Amsterdam, 147-184. crossref

Likar, K.; Jevsnik, M. (2006) “Cold Chain Maintaining in Food Trade”. Food Control, v. 17, pp. $108-113$. crossref

M.h, Hoang, M.h. O Laguerre, J Moureh, D Flicket al. Heat transfer modelling in a ventilated cavity loaded with food product: Application to a refrigerated vehicle. Journal of Food Engineering, [s.I.], v. 113, n. 3, p.389-398, dez. 2012. Elsevier BV. crossref

Moresco, Gustavo Henrique; Silva, Vanina Macowski Durski. Cadeia do Frio: estudo de Roteirização e Performance Térmica Durante a Distribuição de Alimentos Congelados. Encontro Nacional de Engenharia de Produção. Joinville, p. 1-13. out. 2017.

Novaes, A.G.; Frazzon, E.M., and Burin, P.J. (2010). Dynamic Routing in Over Congested Urban Areas, Proceedings LDIC 2009, Second International Conference on Dynamics in Logistics, Bremen, August, pp. 103-112

Novaes, Antônio Galvão. Logística e gerenciamento da cadeia de distribuição. Rio de Janeiro: Elsevier, 2007.

Pereira, V. F.; Doria, E. C. B.; Carvalho Júnior, B. C.; Neves Filho, L. C.; Silveira Júnior, V. (2010) Avaliação de temperaturas em câmaras frigoríficas de transporte urbano de alimentos resfriados e congelados. Ciência e Tecnologia de Alimentos, Campinas-SP, v. 30, n. 1, p 158-165. crossref 
Recebido: 22 Fev. 2019

Aprovado: 05 Set. 2019

DOl: 10.3895/gi.v15n3.9648

Como citar:

MULLER, G. A.; Silva, V. M. D.. Modelagem Matemática Para a Distribuição Física de Produtos da Cadeia do

Frio. R. Gest. Industr., Ponta Grossa, v. 15, n. 3, p. 185-208, Jul./Set. 2019. Disponível em:

http://periodicos.utfpr.edu.br/revistagi. Acesso em:

Correspondência:

Gabriel Alexandre Müller

Rua Eleotério Maia, número 281, Bairro Guanabara, Joinville, SC, Brasil.

Direito autoral: Este artigo está licenciado sob os termos da Licença Creative Commons-Atribuição 4.0

Internacional.

(c) (1) 